\title{
Korrespondenzen.
}

\section{Wer hat die Radiumemanation in die 'Therapie eingeführt?}

Von Priv.-Doz. Dr. A. Braunstein in Moskau.

Fast jeden l'ag bringt ıns die medizinische Presse Mitteilungen über die Anwendung von Radiumeınanation bei verschiedenen Krankheiten. Dabei werden leider die ersten Arbeiten über diese Frage nicht nur nicht berïcksichtigt, condern nicht einmal erwähnt. So hat Loe wenthal in seiner ausführlichen Publikation über die Wirkung der Radiunemanation ${ }^{1}$ ), sowie F. Miendel in seiner in No. 3 dieser Wochenschrift erschienenen Arbeit über Emanationstherapie meine Untersuchungen, die auf diesem ('iebiete die ersten waren, nicht einmal erwähnt.

Schon im Jahre 1904 habe ich zuerst Radiumemanation in Form von radioaktivem Wasser und Wismut für therapeutische Zwecke angewandt und habe darïber dann in der Therapie der Gegenwart berichtet. ${ }^{2}$ ) Ich habe schon damals durch Destillation gewonnenes Radiumemanations. wasser in Tumoren gespritzt und dadurch Verflüssigung bösartiger Geschwülste erzielt. Außerdem habe ich zuerst per os radioaktives Wismut Kranken mit Magen- und Speiseröhrenkrebs verabreicht. Die zuerst von inir festgestellten Beobachtungen über die Wirkung der Radiumemanation veranlaßten mich zu weiteren Untersuchungen, die von mir zusammen mit P. Bergell im Berliner Krebsinstitut ausge. führt wurden. Es folgte dann anfangs 1905 eine Publikation ${ }^{3}$ ) von uns in der „Medizinischen Klinik", wo wir mitteilten, daß, während die Bestrahlung mit Radiun die Fermentwirkung des Pankreatins deutlich hemmt, diese Enzymwirkung beträchtlich verstärkt wird unter dem Einfluß von Radiumemanation. Und so habe ich und Bergell zuerst die aktivierende Wirkung der Radiumemanation auf Pankreasferment nachgewiesen. Dieselbe befördernde Wirkung der Emanation auf Fermente haben später Bergell und Bickel auch für die peptische Verdauung bestätigt. Endlich habe ich im April 1905 auf dem 22. Kongreß für innere Medizin in Wiesbaden einen Vortrag über Bedeutung und Anwendung der Radiumemanation gehalten, in welchem ich noch auf verschie. dene andere Gesichtspunkte hingewiesen habe. Ich teilte ${ }^{4}$ ) damals mit da $ß$ erstens durch Verabreichung von radioaktivem Wasser eine durch Milchsäurebazillen verursachte Gärung im Magen zu beseitigen möglich ist, und zweitens, daß die fermentaktivierende Fähigkeit der Radiumemanation auch bei der Therapie der Magen- und Stoffwechselkrankheiten, z. B. Diabetes, init Mineralwasserquellen von größter Bedeutung zu sein scheint.

$\mathrm{Da}$ meine Untersuchungen, die in den drei von mir publizierten Arbeiten erschienen, auf dem Gebiete der Radiumemanation die ersten waren, so glaube ich, daß diese bei der Besprechung der Frage ein Ignorieren seitens einiger Autoren keines Falles verdient haben. Ich hoffe den Beweis geliefert zu haben, daß sowohl die Einsprit. zungen, wie auch die oralen Anwendungen von Radium emanation für die Therapie von mir zuerst vorgeschlagen wurden. 